\title{
Buccal mucosa graft in surgical management of Peyronie's disease: Ultrasound features and clinical outcomes
}

\author{
Andrea Fabiani ${ }^{1}$, Fabrizio Fioretti ${ }^{1}$, Maria Pia Pavia ${ }^{2}$, Luca Lepri $^{1}$, Emanuele Principi ${ }^{1}$, Lucilla Servi $^{1}$ \\ ${ }^{1}$ Urology Unit, Surgical Department, ASUR Marche Area Vasta 3, Macerata, Italy; \\ ${ }^{2}$ Resident, Division of Urology, Marche Polythecnic University, Ancona, Italy.
}

\begin{abstract}
Summary Introduction: Plaque incision and grafting represent the best surgical approach to the Peyronie's Disease (PD). The grafting procedures must be restricted to patients with normal preoperative status, excessive curvature and/or deformities. However, the ideal graft has not been identified yet. Buccal mucosa grafts (BMG) provided excellent short-term results, ensuring the fast return of spontaneous erections and preventing shrinkage, which is the main cause of graft failure. Another fearsome surgical complication is de novo erectile dysfunction (ED). We report our results with BMG focusing on the analysis of ultrasonographic and clinical data demonstrating buccal mucosa as determinant factor that allow to avoid complications.
\end{abstract}

Materials and methods: From 2013 to 2019 we performed at our Urology Unit 27 corporoplasties with BMG to correct complex penile curvature due to PD. Clinical, post-surgical and ultrasound follow up data were evaluated. All patients were no responders to medical treatment or previous surgical procedures. The evaluation period was 72 months. Data regarding pre-operative work-up, including IIEF (IIEF-5) questionnaire administration, detailed clinical history and penile dynamic ultrasound (PGE1-induced erection) were collected. The time of spontaneous erection resumption was recorded for each patient. To improve blood supply to the graft, a low-dose PDE5-i was prescribed for all patients for a period of two months, starting immediately after discharge. Check-ups were scheduled every 3 months, starting from 1 month after surgery. In each visit, patients underwent a penile ultrasound evaluation of graft features. After 6 and 12 months, all patients underwent a penile dynamic ultrasound for Erection Hardness Score determination, then standard ultrasound and clinical evaluation yearly. Our analyses were focused on $B M G$ as a major determinant of the surgical success.

Results: Mean age of 27 patients was 57 years (42-71) with a maximum follow up time of 72 months and minimum of 3 . Site of penile curvature was dorsal in $18(67 \%)$ patients, ventral in $2(7 \%)$, complex in $7(26 \%)$. The degree of the curvature was $<60^{\circ}$ in $11(41 \%)$ patients, $>60^{\circ}$ in $16(59 \%)$. Straightening of penis was reached in $100 \%$ of cases. Penile shortening resulted in $7.4 \%(2 / 27)$. De novo ED appeared in $2 / 27$ cases with a post-operative rate of PDE5i users increasing from 12 to 14 patients (45\% vs $52 \%$ ). Ultrasound aspects of BMG, recorded at every follow up visit, results in a hypoechoic plaque with an iperechoic rim that become isoechoic over the time in all cases. No case of scars or seroma was registered. Small intra-graft cystic lesions were highlighted in 3 cases (11\%).

Conclusions: BMG may represent a good choice in grafts pro- cedures for PD surgical management. The functional results obtained by $B M G$ procedures were related to the good anatomical characteristics of the patch and were highlighted in our series by use of penile ultrasound, during the follow up period.

KEY WORDS: Peyronie's disease; Buccal mucosa; Ultrasound; Graft; Erectile dysfunction.

Submitted 13 January 2021; Accepted 27 January 2021

\section{INTRODUCTION}

Peyronie's disease (PD) is a benign condition causing penile deformities, shortening, loss of penile flexibility and sexual dysfunctions (1). The diagnoses are increasing in number, even in the younger population, because of the increased knowledge about the topic (2). Surgical treatment remains the gold standard and it includes penis plication, grafting, and placement of inflatable prosthesis with the aim to restore coital functions (3). Buccal mucosa grafts (BMG) provided excellent shortterm results, suggested by the early recovery of spontaneous erections and the prevention of late shrinkage, which is the main cause of graft failure. It also seems to be safe and reproducible, thus representing a valuable treatment option for PD. Clinical series are limited yet but functional and cosmetics results are very promising (4). We analyzed our surgical results with the BMG technique applied to 27 patients focusing on the ultrasonographic follow-up and clinical data.

\section{Methods}

From our surgical data base, we evaluated 27 consecutive cases treated with a plaque incision and BMG to correct the secondary penile curvature due to PD. Clinical, postsurgical and ultrasound follow up data were evaluated. All patients were no responders to medical treatment or previous surgical procedures. The evaluation period was 72 months. Data regarding pre-operative work-up, including IIEF (IIEF-5) questionnaire administration, detailed clinical history and penile dynamic ultrasound (PGEl-induced erection) features were collected $(5,6)$. The surgical procedures were done previous penile degloving and using a relaxing incision (double $\mathrm{Y}$ or $\mathrm{H}$ shaped) at the point of maximum curvature on the penis. The albuginea defects were covered using BMG grafting

No conflict of interest declared. 
technique $(7,8)$. The buccal mucosa grafts were harvested as described by Eppley et al. (9). After "defatting", BMG was apposite to cover the albuginea defect with the submucosa surface in contact with the cavernous tissue in order to obtain a quick blood supply and sutured with a 3/0 adsorbable running sutures (Maxon ${ }^{T M}$ ) in each side (Figure 1). Artificial erection was repeated to evaluate the curvature and deformity correction, defining the need of complementary tunica albuginea plications (10). Buck's fascia was accurately closed with interrupted adsorbable suture, especially in correspondence with the patch site to avoid patch enlargement. In cases of ventral curvatures, urethra was fixed to corpora cavernosa with a tension-free stitches. A small drainage was placed between the Buck's fascia and the dartos. Circumcisional incision was closed and dressed. Patients were discharged at third post-operative day after catheter, drainage and dressing removal. The time of spontaneous erection resumption was recorded for each patient. To improve blood supply to the graft, a lowdose PDE-5 inhibitor (PDE-5i) was prescribed for all patients for a period of two months, starting immediately after discharge. Check-ups were scheduled every 3 months, starting from 1 month after surgery. In each visit, they underwent a penile ultrasound evaluation of graft fea-

Table 1.

Salient pre-operative clinical data.

\begin{tabular}{|l|c|}
\hline Number of patients & $\mathbf{2 7}(\mathbf{1 0 0 \% )}$ \\
\hline Age (years) & $57(42-71)$ \\
\hline Penile curvature degree pre-op & $61.9^{\circ}(45-90)$ \\
Duration of PD (months) & $16.5(6-48)$ \\
IIEF score pre -operative & $22.5(18-24)$ \\
\hline Curvature side & Dorsal $18(67 \%)$ \\
& Ventral 2 (7\%) \\
\hline PDE5i use pre-op & Complex 7 (26\%) \\
\hline Diabetes mellitus & $12(45 \%)$ \\
\hline Previous PD surgery & $4(14.8 \%)$ \\
\hline Previous radical prostatectomy & $1(3.8 \%)$ \\
\hline Dupuytren disease associated & $4(14.8 \%)$ \\
\hline Psoriasis & $1(3.7 \%)$ \\
\hline Preputial Lichen Sclerosus & $1(3.7 \%)$ \\
\hline Cardiovascular disease & $2(7.4 \%)$ \\
\hline
\end{tabular}

Table 2.

Results and complications.

\begin{tabular}{|l|c|}
\hline RESULTS & (range; \%) \\
Mean follow up time (months) & $28.3(3-72)$ \\
\hline Time of spontaneous erections resumption (days) & $3.2(1-7)$ \\
IIEF score post operative at last follow up & $23.1(15-24)$ \\
PDE5i post operative use & $14(52 \%)$ \\
Functional penile straightening & $27(100 \%)$ \\
Penile shortening & $2(7.4 \%)$ \\
CoMPLICATIONS & \\
\hline Hemorrage at the donor site & $1(4.7 \%)$ \\
Penile shaft hematoma & $1(4.7 \%)$ \\
Glandular erection pain & $1(3.7 \%)$ \\
Preputial edema & $4(14.8 \%)$ \\
Skin necrosis & $1(3.7 \%)$ \\
Cystic intra-graft lesions & $3(11 \%)$ \\
Penile abscess & $1(3.7 \%)$ \\
Recurvatum relapse & $0(0 \%)$ \\
De novo erectile dysfunction & $2(7.4 \%)$ \\
\hline
\end{tabular}

Figure 1.

Surgical steps for BMG procedures in a case of ventral curvature.

A. Buccal mucosa graft harvesting; B. Idraulic erection after penile degloving; C. Urethral isolation;

$D$. Marked transversal incision (double $Y$ ) on the plaque;

E. Buccal mucosa grafting; F. Final result.

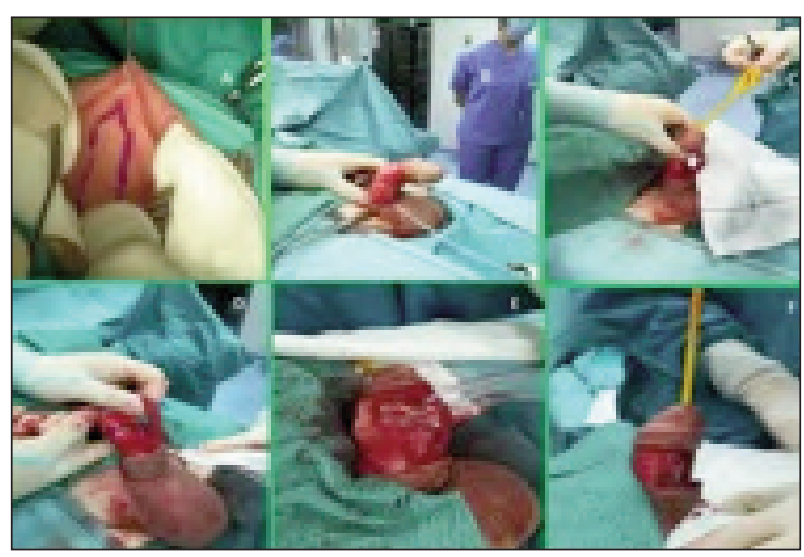

tures. After 6 and 12 months, all patients underwent a penile dynamic ultrasound for Erection Hardness Score determination, then standard ultrasound and clinical evaluation yearly. All consecutive cases who underwent BMG in a 72-month period of time were analyzed. We studied the ultrasonographic appearance of the BMG at 1, 3, 6 and 12 months after surgery and yearly cosmetics and functional outcomes.

\section{Results}

We performed 27 procedures in men with mean penile curvature of degree $61.9^{\circ}$ (range $45^{\circ}-90^{\circ}$ ), mean age 57 years (42-71) and after mean follow-up of 28.3 months ( $\min 3 \max 72$ ). The degree of the curvature was $<60^{\circ}$ in $11(41 \%)$ patients, $>60^{\circ}$ in $16(59 \%)$. Site of penile curvature (degree $61.9^{\circ}$ in mean; range $45^{\circ}-90^{\circ}$ ), was dorsal in $18(67 \%)$ patients, ventral in $2(7 \%)$, complex in 7 (26\%). Pre-operative data and results are summarized in Tables 1, 2. The patient medical history was characterized from different comorbidities recognized as erectile dysfunction (ED) risk factors. Diabetes mellitus was reported in 4 (14.8\%) patients; cardiovascular disease in 2 (7.4\%), previous radical prostatectomy in 4 (14.8\%), preputial lichen sclerosis in 2 (7.4\%) and psoriasis in 1. One patient had a failed plication procedure. Pre-operative use of PDE-5i was referred by 12 patients (45\%). Pre-operative IIEF score was 22.5 in mean (11-24). After a follow up period of 28.3 months (3-72), we reported a functional straightening of penis reached in $100 \%$ of cases. Penile shortening resulted in $7.4 \%(2 / 27)$. All patients had complete spontaneous erections in a period of 1 to 7 days after surgery, however paraphimosis occurred in three cases $(14.2 \%)$, requiring surgical revision, and one patient (4.7\%) develop skin preputial necrosis managed by topical therapy. Post-operative mean IIEF score, at the last follow up was 23.1 (1524). De novo ED appeared in $2 / 27$ cases. Post-operative amount of PDE5i users increasing from 12 to 14 patients (45\% vs 52\%). Ultrasound was applied at each follow-up visit. Ultrasound features during follow up are showed in 
Figure 2.

A. Ultrasound patch (white arrow) appearance 3 months after surgery.
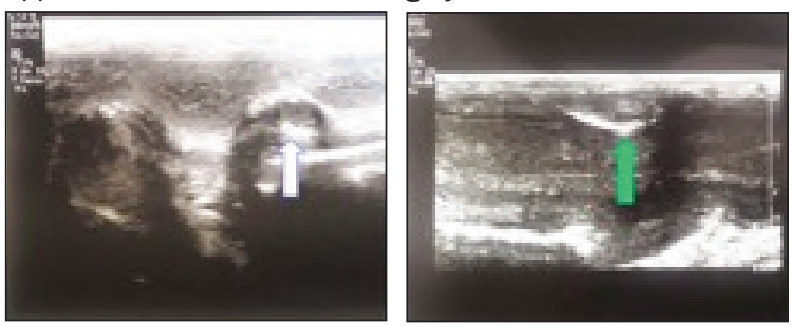

B.

Ultrasound patch (green arrow) appearance after surgery (6 months).

Figure $2 \mathrm{a}, \mathrm{b}$ and Figure 3. In all cases, post-operative images consisted in a hypoechoic plaque with hyperechoic rim, observed in the graft area during the first month after surgery. Progressively, we described the ultrasound disappearance of graft, which has become isoechoic respect the tunica albuginea and not distinguishable from albuginea rim. No case of scars or seroma was registered. Small intragraft cystic lesions highlighted in 3 cases (11\%).

\section{Discussion}

Severe PD $\left(>60^{\circ}\right)$ is still treated by surgical approach that leads an efficient solution in a relatively short time (11). In spite of this, results are not devoid of complications, the most serious is the development of de-novo ED, which seems to be caused by complete surgical excision of the plaque (12). Nowadays, plaque incision surgery with grafting is the preferred therapeutic approach $(3,8)$.

In 2005, buccal mucosa was introduced as free autograft in the surgical treatment of PD (13) and then, during the

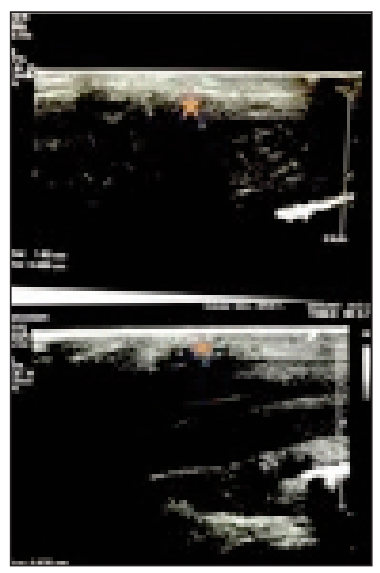

Figure 3.

Complete patch disappearance in ultrasound evaluation (star; 6 years after surgery).

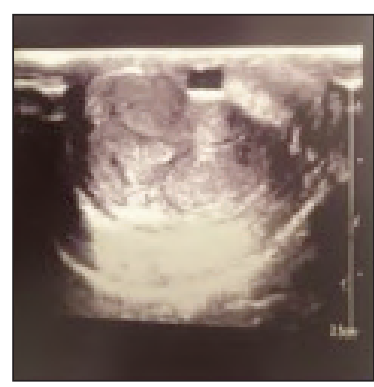

Figure 4.

Small intra-patch cystic lesion. years, evaluated in several others series (14-19). Promising results were reported by Shioshvili et al. (13) who observed the complete straightening of penis in $92.3 \%$ of 26 patients treated with a residual curvature $\left(<10^{\circ}\right)$ in $7.7 \%$. The shortening of penis was observed in four patients (15.4\%) and in two patients (7.7\%) a partial reduction of erectile function. They concluded that buccal mucosa showed high properties of adaptation and revascularization, good anatomical and functional clinical results, demonstrating a stable elasticity without late shrinkage. Similarly, Liu et al. (14) showed that buccal mucosa remained stable with good elasticity over time. They performed replacing the plaque by free autograft of buccal mucosa on 24 patients and obtained satisfactory results: complete penile straightening was achieved in 21/24, slight residual curvature (< 15 degrees) noted in 3 (12.5\%), a little shortening of the penis $(<1 \mathrm{~cm})$ in 2 $(8.3 \%)$. As advancement in term of prevention of de novo ED, Cormio and co-workers (15) introduced important technical modifications consisting in the plaque incision instead of excision. BMG was performed similar than others series. In 15 consecutive surgical procedures they reported a $100 \%$ of penile straightening, no curvature recurrence and any de novo ED. These results were confirmed by Zucchi et al. (16) in 32 patients treated with plaque incision and BMG between 2006 and 2013: in 28 cases, they reported one curvature relapse after 1 year (3.5\%) and an immediate significant improvement of IIEF scores in half of cases, although after 2 years the trend was in reduction. They concluded that corporoplasty with buccal mucosa represent a good treatment choice for most forms of PD. The complete penis straightening was achieved in $73 \%$ of 33 patients by Gvasalia et al. (17), however a residual curvature $\left(<20^{\circ}\right)$ was observed in $27 \%$ and de novo ED in $18 \%$, commonly in elderly patients, but with a good response to PDE-5i. Molina-Escudero et al. (18) reported the short-term results (penile length, angle of curvature and erectile function) of 10 patients treated through corporoplasty with oral mucosa graft. The straightening of penis was reached in $100 \%$ with a shortening rate of $80 \%$ and $10 \%$ of de novo ED. In our previous experience with 17 cases (19), we reported favorable results. In this series, that take account of 10 more patients passing from 17 to 27 cases, we have similar results with a longer follow-up. All the procedures were performed as the main indication the failure or inability to sexual intercourse and psychological impairment due to the penile curvature. Patients are variably aging, with a medical general history characterized from different comorbidities as, in example, diabetes mellitus, cardiovascular disease, previous pelvic surgical procedure for prostate cancer (Table 1). One patient underwent a previous failed plication procedure. Pre-operative use of PDE5 inhibitors was referred by 12 patients (45\%). Recognized that several patients had more risk factors of post-operative ED (baseline sexual function, age, graft size or position) (12), the probability of de novo ED should be higher, than that observed. We obtained a very good functional results with $7.4 \%$ of de novo ED and a post-operative increase of PDE5i users to $52 \%$ from $45 \%$. In literature the rate of de novo ED ranges between $0 \%$ to $18 \%$. These results may be explained by the nature of buccal mucosa that is a living 
tissue with an elevated binding capacity and revascularization which is immediately supplied with blood from the cavernous tissue. In this way, buccal mucosa tends to heal rapidly, immediately integrating with the surrounding albuginea tissue. This translates into a more rapid resumption of spontaneous erections (mean of 3,26 days in our experience) and sexual activity and into a reduced risk of curvature relapse (20). Penile ultrasound validated these results showing the process of mucosal patch integration with tunica albuginea. Ultrasound has been showed to be a method of choice because his cost effectiveness, repeatability and low invasiveness, if compared to others methods (ie. Magnetic Resonance) (21). US is mainly used in diagnosis and staging of PD. There are only few reports regarding the ultrasound usefulness in the follow up after treatment. In relapses or in dilations patch evaluation, ultrasound is still decisive (22). In our series, US showed that BMG appears initially as hypoechoic plaques with hyperechoic rim (Figure 2a, b) and then evolved in isoechoic over the time. After six years from surgical procedure, we reported a complete disappearance of the patch aspect, confounded with the albuginea (Figure 3). No cases of scars, and consequently, of curvature relapse, or seroma were registered. Small intra-graft cystic lesions highlighted in 3 cases (11\%) (Figure 4). Any patch bulging has been reported yet.The perfect seal of BMG and the accurate closure of Buck's fascia are important key-factors to obtain a successful result. The biologic characteristics of buccal mucosa reduced the risk of curvature relapse as demonstrated by the high rate of penile straightening reported in $100 \%$ in our series while is $87.5-100 \%$ in literature. Patch ultrasound evaluation confirm these aspects and it is needed in the surgical management of PD.

\section{Conclusions}

The functional results obtained by BMG procedures in PD surgical treatment were related to the great anatomical characteristics of the patch and highlighted in our series by use of penile ultrasound during the follow up period. Ultrasound features confirmed the characteristics of buccal mucosa as perfect seal in the tunica albuginea defect, created after fibrotic plaque incision, allowing to reduce the risk of de novo ED.

\section{REFERENCES}

1. Hatzimouratidis $K$, et al. EAU Giudelines on Erectile dysfunction, Premature Ejaculation, Penile curvature and Priapism. 2018.

2. Paulis G., Cavallini G., Barletta D. et al. Clinical and epidemiological characteristics of young patients with Peyronie's disease: a retrospective study. Res Rep Urol. 2015; 7:107-111.

3. Hatzimouratidis K, Hatzichristou DG. Plaque incision and grafting represents the best surgical approach to Peyronie's Disease patient: Con. Current Sexual Health Reports. 2006; 3:56-60.

4. Garcia Gomez B, Ralph D, Levine L, et al. Grafts for Peyronie's disease: a comprehensive review. Andrology. 2017; 6:117-126.

5. Martino P, Galosi AB, Bitelli M, et al. Practical recommendations for performing ultrasound scanning in the urological and andrological fields.Arch Ital Urol Androl. 2014; 86:56-78.

6. Dell'Atti L, Galosi AB. Ultrasound findings of ruptured Peyronie's plaque: Case report and review of the literature. Arch Ital Urol Androl. 2017; 89:85-86.

7. Dell'Atti L, Polito M, Galosi AB. Is Degloving the Best Method to Approach the Penile Corporoplasty With Yachia's Technique? Urology. 2019; 126:204-208.

8. Brant WO, Bella AJ, Garcia MM, et al. Correction of Peyronie's disease: plaque incision and grafting. BJU Int. 2006; 97:1353-60.

9. Eppley BL, Keating M, Rink R. A buccal mucosal harvesting technique for urethral reconstruction. J Urol. 1997; 157:1268.

10. Dell'Atti L, Scarcella S, Tallè M, et al. Simultaneous curvature correction at the time of the penile fracture repair: surgical and functional outcomes. Res Rep Urol. 2019; 11:105-110.

11. Mulhall J, Anderson M, Parker M. A surgical algorithm for men with combined Peyronie's disease and erectile dysfunction: functional and satisfaction outcomes. J Sex Med. 2005; 2:132-138.

12. Flores S, Choi JM, Alex B, et al. Erectile dysfunction after plaque incision and grafting: short term incidence and predictors. J Sex Med. 2011; 8:2031-37

13. Shioshvili TJ, Kakonashivili AP. The surgical treatment of Peyronie's disease: replacement of plaque by free autograft of buccal mucosa. Eur Urol. 2005; 48:129-35

14. Liu B, Zhu XW, Zhong DC, et al. Replacement of plaque by buccal mucosa in the treatment of Peyronie's disease: a report of 27 cases Zhonghua Nan Ke Xue. 2009; 15:45-7.

15. Cormio L, Zucchi A, Lorusso F, et al. Surgical treatment of Peyronie's disease by plaque incision and grafting with buccal mucosa. Eur Urol 2009; 55:1469-1475.

16. Zucchi A, Silvani M, Pastore AL, et al.Corporoplasty using buccal mucosa graft in Peyronie disease: is it a first choice? Urology. 2015; 85:679-83.

17. Gvasalia B, Kochetov A, Abramov R, et al. Buccal mucosa in the surgical treatment of Peyronie's disease. J Sex Med. 2014; 11 (Suppl 1):33.

18. Molina Escudero R, Alvarez-Ardura M, Redon-Galvez L, et al. Cavernoplastia con injerto de mucosa oral para el tratamiento quirurgico de la enfermedad de La peyronie. Actas Urologicas Espanolas. 2016; 40:328-332.

19. Fabiani A, Servi L, Fioretti F, et al. Buccal mucosa is a promising graft in Peyronie's disease surgery. Our experience and a brief literature review on autologous grafting materials. Arch Ital Urol Androl. 2016; 88:115-121

20. Costantini E, Zucchi A. Reconstructive surgery in Peyronie's disease: What's new? World J Clin Urol. 2015; 4:1-4.

21. Dell'Atti S, Manno S, Scarcella, et al. Analysis of penile ultrasound in Peyronie Disease: Variability and interpretation of sonographic patterns.European Urology Supplements. 2018; 17:e2751.

22. Rolle L, Tamagnone A, Bollito E, et al. Could plaque excision surgery with sis graft induce a new fibrotic reaction in la Peyronie's disease patient? Arch Ital Urol Androl. 2007; 79:167-169.

\section{Correspondence}

Andrea Fabiani, MD (Corresponding Author) andreadocl@libero.it

Fabrizio Fioretti, MD - fa.fioretti@libero.it

Lepri Luca, MD - luca.lepri@sanita.marche.it

Emanuele Principi, MD - emanuele.principi@sanita.marche.it

Lucilla Servi, MD - lucilla.servi@sanita.marche.it

Urology Unit, Surgical Dpt, ASUR Marche Area Vasta 3, Macerata (Italy)

Maria Pia Pavia, MD - mariapia.pavia@gmail.com

Resident, Division of Urology, Marche Polythecnic University, Ancona (Italy) 